\title{
Fast Restoration of a Broad-Spectrum SARS-Cov Therapeutic Antibody for SARS-Cov-2
}

\author{
Sara Zhang, Mike Young and Jason J. Pan*
}

Structural Characterization Division, NovoAb Bioanalytics, Victoria, British Columbia, Canada V8P 2L5

\section{* Corresponding author:}

Jason J. Pan, Ph.D.

Structural Characterization Division

NovoAb Bioanalytics, Inc.

1095 McKenzie Ave.

Victoria BC, Canada V8P 2L5

Email: jpan@novoab.com

Tel: (778) 588-1244

Fax: (250) 704-4440 


\begin{abstract}
:
The rapid spread of SARS-Cov-2 remains a major threat for public health and global economy, both preventative and therapeutic solutions are therefore urgently needed. Through the use of epitope-guided antibody design, we successfully restored a broad-spectrum SARS-Cov therapeutic antibody for SARSCov-2. Compared to the precursor antibody CR3022, the newly designed antibody NOVOAB-20 binds to SARA-Cov-2 receptor binding domain (RBD) with a more than 10-fold higher affinity. Because this antibody targets a highly conserved epitope and the mutations on SARS-Cov-2 known so far are all not in this region, it also has the potential to block future SARA-Cov-2 mutants. As a fully humanized antibody, NOVOAB-20 is a promising candidate to be developed as potential therapeutics for SARS-Cov-2, either as monotherapy or in combination with other neutralizing antibodies targeting different epitopes (e.g. the ACE2 binding site). This fast antibody restoration rationale may also be useful for designing drugs for other pandemic-causing viruses.
\end{abstract}


Effective therapeutics against SARS-Cov-2 is urgently needed to contain the ongoing global COVID-19 pandemic. Aside from vaccine development, therapeutic monoclonal antibody $(\mathrm{mAb})$ is considered one of the most promising alternative approaches as it potentially can not only prevent but also treat the disease, and may work better for the elderly or people who are immune compromised. SARS-Cov-2 uses its receptor binding domain (RBD) on the S protein to engage the host receptor ACE2 for viral entry. ${ }^{1,2}$ RBD-targeting antibodies that blocks ACE2 binding could neutralize the virus. A number of such mAbs have been discovered from the blood of recovered COVID-19 patients. ${ }^{3-7}$ However, since the ACE2 binding site (epitope) on RBD mutates rather quickly, mAbs targeting this site may easily lose activity for new viral mutants. This is actually what happened for SARS-Cov 17 years ago - all of the effective mAbs developed for SARS-Cov lost their neutralizing ability on SARS-Cov-2. ${ }^{8,9}$ When developing a mAb drug against rapidly mutating viruses like SARS-Cov-2, it is desirable if the drug works not only for the original virus, but also for its mutated variants. This is the focus of the current work.

CR3022 is a unique broadly neutralizing antibody discovered for SARS-Cov, and it targets a highly conserved cryptic epitope on RBD. ${ }^{9,10}$ It completely blocked all mutant escapes of CR3014, a mAb that targets ACE2 interaction site on SARS-Cov RBD. ${ }^{10}$ However, CR3022 lost neutralization ability for SARS-Cov-2, although it still binds to the viral RBD. ${ }^{9}$ Considering the extremely high similarities between SARS-Cov and SARS-Cov-2 (almost identical 3D structure of their RBDs and Spike proteins; both viruses use the same receptor ACE2 for cell entry ; and the binding modes between the two RBDs and ACE2 are nearly identical), ${ }^{1,2,8,11}$ we reasoned that the diminished neutralization of CR3022 could be due to its more than 10-fold lowered binding affinity to SARS-Cov-2 RBD compared to SARS-Cov.,10 This motivated us to design a cross-reactive antibody for SARS-Cov-2 based on CR3022 and its epitope. The workflow is depicted in Scheme 1. 
Scheme 1. Workflow of epitope-guided antibody design (EGAD).

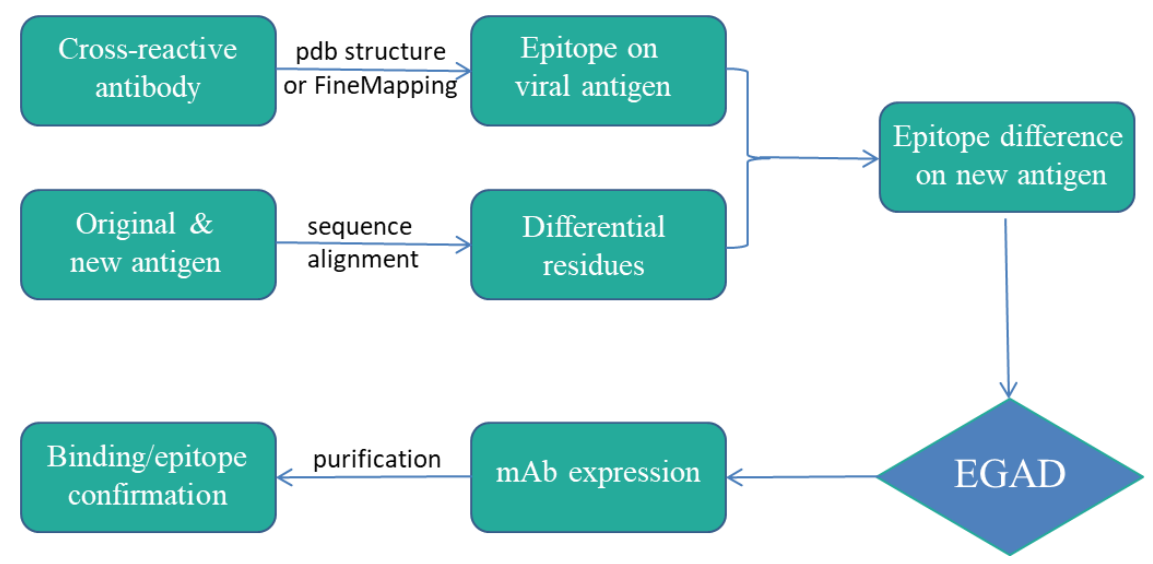

In the epitope-guided antibody design (EGAD) workflow, the original cross-reactive antibody is CR3022, and the original and new antigen is RBD of SARS-Cov and SARS-Cov-2, respectively. CR3022's epitope ${ }^{9}$ on SARS-Cov-2 RBD antigen is shown in Figure 1A. It is clear that this mAb binds to a location away from the ACE2 epitope. ${ }^{2}$ The differential residues between SRAS-Cov RBD and SARS-Cov-2 RBD derived from sequence alignment are displayed in Figure 1B (Magenta), together with newly mutated residues (Red) for SARS-Cov-2. ${ }^{12}$ By comparing Figure 1A and B, we can see that ACE2 binding epitope is located in the hot spot of mutations, while the epitope of CR3022 in a highly conserved region with much less mutations. By mapping the mutated amino acids onto CR3022 epitope (Figure 1C), it was found that there are four residues in the epitope that have changed when SARS-Cov evolved into SARS-Cov-2. These residues (A372, P384, T430, and H519 in SARS-Cov-2 numbering) may play a key role in the binding affinity change of CR3022 and are thus the focus of our antibody design. 


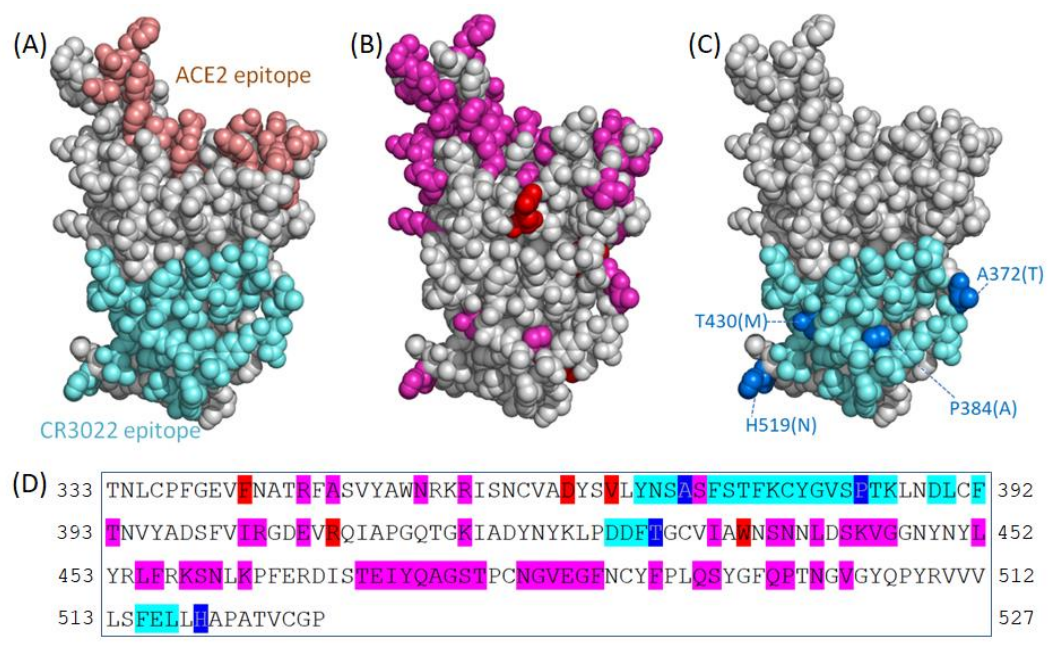

Figure 1. Global view of the epitopes and mutations on SARS-Cov-2 RBD structure (pdb entry 6w41). (A) Location of CR3022 epitope (Cyan) ${ }^{9}$ and ACE2 epitope (Orange) ${ }^{2}$ (B) Differential residues between SRAS-Cov RBD and SARS-Cov-2 RBD (Magenta), and newly mutated residues in SARS-Cov-2 RBD (Red); (C) Location of differential residues (Blue) within the CR3022 epitope (Cyan); (D) View of the CR3022 epitope and mutated residues in SARS-

Cov-2 RBD sequence.

Multiple factors are considered in our EGAD program to optimize the interaction of new antibody candidates with the SARS-Cov-2 RBD antigen. These include amino acid hydrophobic interactions, electrostatic interactions, aromatic-aromatic interactions, hydrogen bonding of both main chain and side chain, backbone-dependent bond angles, potential new salt bridges, and side chain-solvent interactions. When we perform in silico amino acid mutation in the precursor antibody, CR3022 in this case, the potential effect on the conformational dynamics of the corresponding antibody-antigen complex is also taken into account based on our extensive experience in antibody epitope mapping. Here we take epitope residue H519 as an example. In the crystal structure of CR3022-RBD (SARS-Cov-2) complex, ${ }^{9}$ this histidine interacts with N29 on the light chain of CR3022 (Figure 2B). However, in SARS-Cov RBD, for which CR3022 was initially developed, the amino acid at this position was an asparagine (N505, Figure 2A). To strengthen the interaction with H519 of SARS-Cov-2 RBD, N29 on the antibody can be replaced by aspartic acid to form a possible salt bridge with protonated histidine, or by phenylalanine to form a 
more favorable His-Phe interaction as indicated in computer modeling (Figure 2C). ${ }^{13}$ Tryptophan, lysine, tyrosine and glutamic acid were also considered at this position.

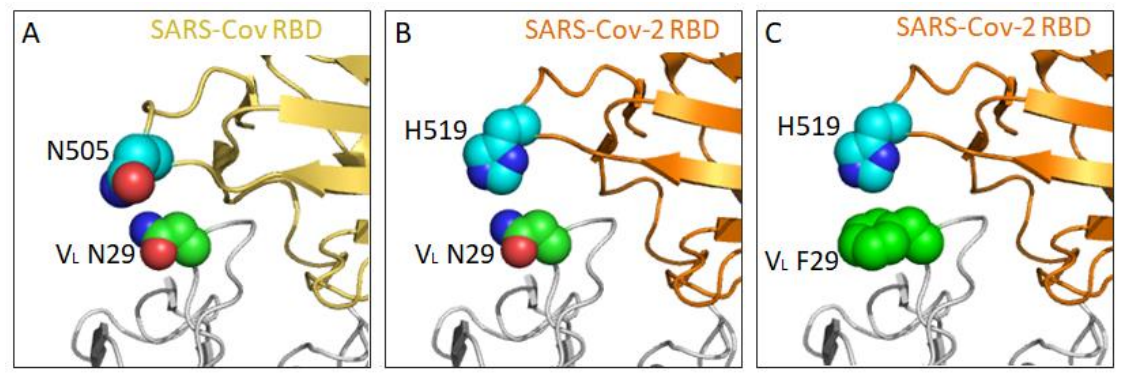

Figure 2. Interaction between antibody light chain residue 29 and RBD. (A) N29 of CR3022 with N505 of SARSCov RBD; (B) N29 of CR3022 with H519 of SARS-Cov-2 RBD (pdb entry 6w41); (C) F29 of newly designed mAb candidate NOVOAB-6 with H519 on SARS-Cov-2 RBD.

After in silico mutagenesis mimicking natural antibody somatic mutation and affinity maturation, another step of EGAD workflow is antibody humanization, in order to minimize potential immunogenic effects. Because the original CR3022 is a fully human antibody, the new mAb candidates without change in the framework do not need humanization in these regions. Mutations in the CDRs (complementaritydetermining regions) were checked by homology search using publicly accessible database IgBlast and our own antibody databases. In a hope of helping combat the severely ongoing COVID-19 pandemic as quickly as possible, we decided to go ahead and produce the top mAb candidate we designed, which is NOVOAB-20. This mAb was successfully expressed in mammalian cells and purified to $95 \%$ purity, and it turned out to be stable in solution even at high concentrations (> 5mg/ml). Its binding ability was measured by ELISA (enzyme-linked immunosorbent assay) experiments using SARS-Cov-2 RBD as the immobilized antigen (Figure 3). The binding EC50 value of NOVOAB-20 in full IgG form was found to be $19 \mathrm{ng} / \mathrm{ml}$, which equals to $0.126 \mathrm{nM}$. This is a 16 -fold increase in binding affinity compared to its precursor mAb CR3022 $(2.1 \mathrm{nM}),{ }^{8}$ indicating the success of our design. This binding affinity is also comparable to that of CR3022 binding to SARS-Cov RBD. ${ }^{10}$ 


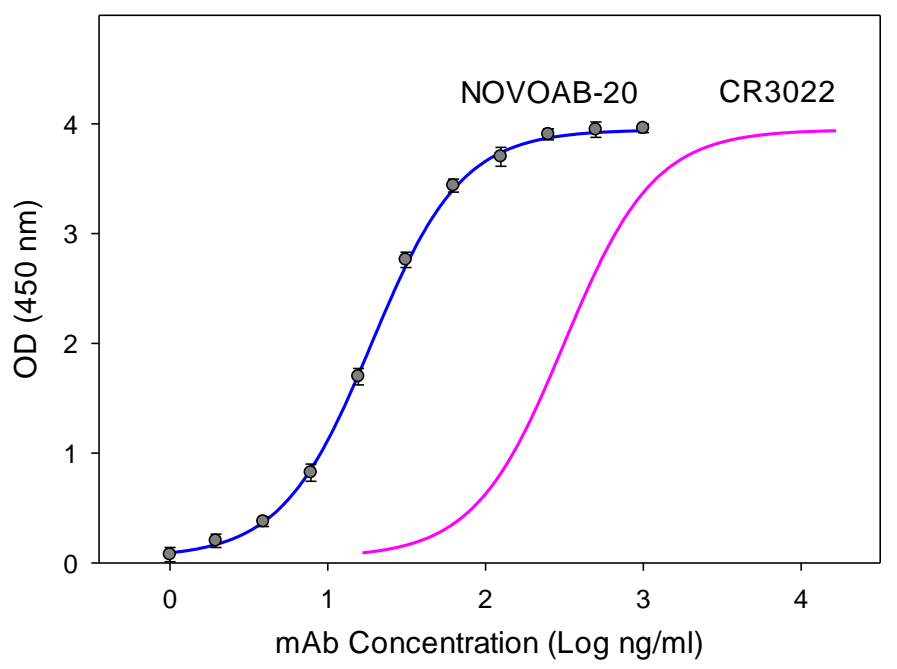

Figure 3. ELISA curves of antibody binding to SARS-Cov-2 RBD: NOVOAB-20 (Blue), CR3022 (Magenta).

In summary, we successfully designed and generated a fully humanized IgG antibody against SARSCov-2 RBD by using epitope-guided antibody design. It has a high binding affinity comparable to the binding between mAb CR3022 and SARS-Cov RBD, which resulted in complete neutralization of the SARS-Cov virus. We expect NOVOAB-20 to work similarly on the current SARS-Cov-2 virus. Since it targets a highly conserved cryptic epitope, it should also work for SARS-Cov-2 mutants. It is worth noting that the mutations on SARS-Cov-2 known so far are all not in the epitope of this mAb. ${ }^{12}$ In addition, CR3022 was reported to exhibit a superior synergy effect when used in combination with CR3014, a mAb targeting the ACE2 binding site on SARS-Cov. ${ }^{10}$ The combination enhanced the potency of both mAbs by 5 and 20 times, respectively. That is a 5-20 times smaller dosage. It would be interesting to test and see if NOVOAB-20 has a similar synergy effect with other COVID-19 mAbs that targeting a different epitope on SARS-Cov-2 (e.g. the ACE2 binding site). As antibody drugs are usually very expensive because of their high production cost, the dramatically (>10x) reduced dosage may be an important factor to consider if antibody drugs are going to be used for COVID-19 patients. It would be also interesting to screen the antibody pools isolated from recovered COVID-19 patients or vaccinated animals through trageted epitope mapping towards this highly conserved epitope, in the hope of 
discovering more cross-reactive mAbs like NOVOAB-20. Compared to the effective but time and labourintensive single B cell sorting method, the fast antibody restoration rationale presented in this work may also be useful for designing drugs for other viruses, especially during a pandemic.

\section{Acknowledgments}

Antibody and antibody sequences are available for research purposes only under an MTA (inquiry contact: Lisa Chang at NovoAb Bioanalytics Inc., cs@ novoab.com), which allows the use of the antibody sequences for non-commercial purposes but without disclosure to third parties. 


\section{REFERENCES}

1. Yan, R., et al. Structural basis for the recognition of SARS-CoV-2 by full-length human ACE2. Science (New York, N.Y.) 367, 1444-1448 (2020).

2. Lan, J., et al. Structure of the SARS-CoV-2 spike receptor-binding domain bound to the ACE2 receptor. Nature 581, 215-220 (2020).

3. Hansen, J., et al. Studies in humanized mice and convalescent humans yield a SARS-CoV-2 antibody cocktail. Science (New York, N.Y.) 369, 1010-1014 (2020).

4. Ju, B., et al. Human neutralizing antibodies elicited by SARS-CoV-2 infection. Nature 584, 115119 (2020).

5. Shi, R., et al. A human neutralizing antibody targets the receptor-binding site of SARS-CoV-2. Nature 584, 120-124 (2020).

6. Wec, A.Z., et al. Broad neutralization of SARS-related viruses by human monoclonal antibodies. Science (New York, N.Y.) 369, 731-736 (2020).

7. Wu, Y., et al. A noncompeting pair of human neutralizing antibodies block COVID-19 virus binding to its receptor ACE2. Science (New York, N.Y.) 368, 1274-1278 (2020).

8. Tian, X., et al. Potent binding of 2019 novel coronavirus spike protein by a SARS coronavirusspecific human monoclonal antibody. Emerging microbes \& infections 9, 382-385 (2020).

9. Yuan, M., et al. A highly conserved cryptic epitope in the receptor binding domains of SARSCoV-2 and SARS-CoV. Science (New York, N.Y.) 368, 630-633 (2020).

10. ter Meulen, J., et al. Human monoclonal antibody combination against SARS coronavirus: synergy and coverage of escape mutants. PLoS medicine 3, e237 (2006).

11. Wrapp, D., et al. Cryo-EM structure of the 2019-nCoV spike in the prefusion conformation. Science (New York, N.Y.) 367, 1260-1263 (2020). 
12. Ou, J., et al. RBD mutations from circulating SARS-CoV-2 strains enhance the structure stability and infectivity of the spike protein.

https://www.biorxiv.org/content/10.1101/2020.03.15.991844v1 (2020).

13. Trachsel, M.A., et al. Modeling the Histidine-Phenylalanine Interaction: The $\mathrm{NH}^{\cdots} \pi$ Hydrogen Bond of Imidazole·Benzene. The journal of physical chemistry. B 119, 7778-7790 (2015). 